\title{
Ärztemangel?
}

\section{Wir sollten auch von den Überkapazitäten sprechen!}

Wir haben 3620 niedergelassene Ärzte zu wenig, hat die Kassenärztliche Bundesvereinigung (KBV) errechnet. Vor allem an Hausärzten und Psychotherapeuten mangele es, so die KBV. Man müsse daher den Arztberuf attraktiver machen. Auch der Patientenbeauftragte der Bundesregierung, Dr. med. Wolfgang Zöller, hat sich in diesem Sinn geäußert. Der Verband der Ersatzkassen (vdek) sieht aber nicht nur einen Ärztemangel, sondern regional auch Überkapazitäten:

- Der Verband der Ersatzkassen (vdek) hat die pauschalen Äußerungen der Kassenärztlichen Bundesvereinigung (KBV) zum Ärztemangel zurückgewiesen. „Es gibt keinen generellen Ärztemangel, sondern höchstens regionale Engpässe, die zu beheben sind“,

\section{Erratum}

Das sind keine Borrelien

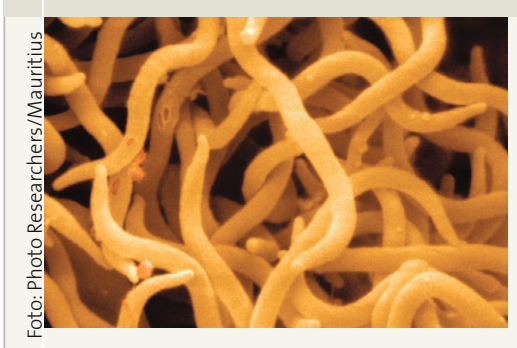

In MMW Nr. 49-50/2009, S. 25 haben wir uns in einer Abbildung vertan, wie einem aufmerksamen Leser nicht entgangen ist:

Das Bild auf Seite 25 in dem Artikel "Chronische Lymeborreliose“ zeigt keine Borrelien, sondern ein Knäuel Spulwürmer. Bei Borrelien wäre die Angabe der Vergrößerung und Färbung eine zusätzliche Information.

- Dr. med. Gerhard Kuhlmann, Humboldtstraße 28, D-32052 Herford erklärte Thomas Ballast, Vorstandsvorsitzender des vdek. Hier seien vor allem die KBV und die Kassenärztlichen Vereinigungen in den Ländern gefordert, gemeinsam mit den Krankenkassen intelligente Lösungsvorschläge zu entwickeln und die ländlichen Regionen für die Ärzte attraktiv zu machen. „Wer von Unterversorgung redet, sollte auch von den Überkapazitäten vor allem in den Städten reden und die Patienten nicht verunsichern."

Die Zahl der ambulant tätigen Ärzte ist in den letzten 20 Jahren um 50\% gestiegen (von 92289 im Jahr 1990 auf 137538 im Jahr 2007). 89\% aller Planungskreise sind überversorgt, 10\% sind regelversorgt. Die meisten Planungskreise sind für Neuniederlassungen gesperrt. In weniger als einem Prozent aller Planungskreise wurde ei- ne bestehende oder unmittelbar drohende Unterversorgung festgestellt.

Vor diesem Hintergrund ist es unverantwortlich, von generellen Engpässen zu sprechen. Vielmehr müssen Überkapazitäten auf der einen Seite abgebaut und in schlechter versorgte Gebiete umgeleitet werden. Bereits heute sind entsprechende Steuerungsinstrumente wie z.B. Zuschläge für unterversorgte und Abschläge für überversorgte Gebiete im Einheitlichen Bewertungsmaßstab vorhanden. Diese differenzierten Punktwerte müssten kurzfristig umgesetzt werden. Übergangsfristen, wie die KBV sie fordert, sind nicht zielführend.

- Michaela Gottfried, Verband der Ersatzkassen e. V., Askanischer Platz 1, D-10963 Berlin

\section{Schweinegrippe-Abrechnung}

\section{Meine KV will nichts von der $\mathbf{8 8 2 0 0}$ wissen}

Ärztliche Leistungen im Zusammenhang mit der Behandlung von Schweinegrippepatienten sollten mit der Pseudo-EBM-Ziffer $\mathbf{8 8 2 0 0}$ gekennzeichnet werden, wie wir mehrfach berichteten. Dies sollte eine Honorierung außerhalb der Regelleistungsvolumina gewährleisten. Offensichtlich haben hier nicht alle KVen mitgespielt:

- Auch in Westfalen-Lippe will die KVWL nichts von der 88200 wissen. Die durch das Robert Koch-Institut berichtete Inzidenz von Influenza mit
Spitzenwerten von 600/100 000 Einwohnern (Epidemiologisches Bulletin Heft 2009;(50):515) soll von den Versorgern in den Regelleistungsvolumina verpackt werden. Die Influenza drängt die Kranken mit den als quälend erlebten Symptomen zum Arzt. Die Versorgung wurde zweckmäßig, und preisgünstig umgesetzt und unsere Körperschaft öffentlichen Rechtes hat nicht den Mumm, von den Kassen Mehraufwand einzufordern.

\footnotetext{
- Dr. med. Martin P. Wedig, Roonstraße 86, D-44628 Herne
} 